\title{
Prolonged breast-feeding protects mothers from later-life obesity and related cardio-metabolic disorders
}

\author{
Petri Wiklund', Leiting Xu' ${ }^{1}$, Arja Lyytikäinen ${ }^{1,2}$, Juha Saltevo², Qin Wang ${ }^{1,3}$, \\ Eszter Völgyi ${ }^{1}$, Eveliina Munukka ${ }^{1}$, Shumei Cheng ${ }^{1}$, Markku Alen ${ }^{4}$, \\ Sirkka Keinänen-Kiukaanniemi ${ }^{5}$ and Sulin Cheng ${ }^{1,6, *}$ \\ 'Department of Health Sciences, University of Jyväskylä, Rautpohjankatu 8, PO Box 35, LL-122, 40700, \\ Jyväskylä, Finland: ${ }^{2}$ Central Hospital, Central Finland, Jyväskylä, Finland: ${ }^{3}$ Department of Endocrinology, \\ West China Hospital of Sichuan University, Sichuan, People's Republic of China: ${ }^{4}$ Department of Medical \\ Rehabilitation, Oulu University Hospital, Oulu, Finland: ${ }^{5}$ Institute of Health Sciences, University of Oulu, Oulu, \\ Finland: 'Department of Orthopaedics and Traumatology, Kuopio University Hospital, Kuopio, Finland
}

Submitted 14 December 2010: Accepted 8 July 2011: First published online 23 August 2011

\begin{abstract}
Objective: To investigate the long-term effects of duration of postpartum lactation on maternal body composition and risk for cardio-metabolic disorders in later life. Design: Retrospective study. Body composition was measured using dual-energy $\mathrm{X}$-ray absorptiometry and serum glucose, insulin and lipids were analysed using enzymatic photometric methods 16-20 years after the last pregnancy. Medical history and lifestyle factors were collected via a self-administered questionnaire. Detailed information regarding weight change patterns during each pregnancy was obtained from personal maternity tracking records.

Setting: City of Jyväskylä and surroundings in Central Finland.

Subjects: Two hundred and twelve women (mean age 48, range 36-60 years). Results: At 16-20 years after their last pregnancy, women who had breast-fed for less than 6 months had higher total body fat mass and fat mass percentage, particularly in the android region $(46 \cdot 5(\mathrm{SD} 8 \cdot 2) \%)$ than mothers who had breast-fed for longer than 6 months $(39 \cdot 0$ (SD 10.2)\%) or for longer than 10 months $(38 \cdot 4$ (sD 10.9) \%, $P<0 \cdot 01$ ). These differences were independent of pre-pregnancy weight and BMI, menopausal status, smoking status, level of education, participation in past and present leisure-time physical activity, and current dietary energy intake. Higher body fat mass was also associated with higher fasting serum glucose concentration and insulin resistance, TAG, LDL cholesterol and total cholesterol concentrations, as well as higher systolic and diastolic blood pressure $(P<0 \cdot 05$ for all).

Conclusions: Short duration of breast-feeding may induce weight retention and fat mass accumulation, resulting in increased risk of cardio-metabolic disorders in later life.
\end{abstract}

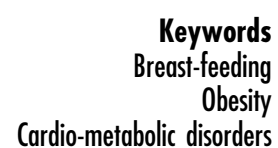

Keywords

Obesity

Cardio-metabolic disorders
Pregnancy is associated with excessive weight gain and central fat deposition $^{(1)}$, which are suggested to serve as an energy reservoir to ensure adequate nutrient supply for the infant $^{(2)}$. Although the increase in maternal fat mass may be essential for the infant, it is evident that pregnancy poses a considerable risk to the mother's health, as manifested by increased blood pressure ${ }^{(3)}$, atherogenic lipid profile and insulin resistance ${ }^{(4)}$. Fortunately, postpartum lactation enables women to reverse this detrimental trend; during lactation, fat is mobilized primarily from the trunk and thighs $^{(5)}$, reducing central fat accumulation, which is considered a major risk factor for metabolic disorders ${ }^{(6)}$.

In spite of the recommendations to breast-feed ${ }^{(7)}$ and the known beneficial effects of breast-feeding on both infant and maternal health, only 18\% of mothers in Finland breast-feed until 12 months ${ }^{(8)}$. In the USA $^{(9)}$ and Europe ${ }^{(10)}$ the trends are similar, indicating that more information about the effects of breast-feeding on maternal health is called for. Animal studies have already suggested that repeated pregnancies without lactation may predispose mothers to obesity $^{(11,12)}$. In humans, however, the weightreducing effects of postpartum lactation have remained controversial. Some studies have shown that breast-feeding significantly reduces weight ${ }^{(13-15)}$, whereas others have reported no effect on maternal anthropometrics and body composition $^{(16,17)}$. These discrepancies in research results may be due to inconsistency in descriptions of breastfeeding patterns, variation in follow-up durations and parity. 
However, given the increasing trend towards overweight and obesity in all developed countries ${ }^{(18,19)}$, and the consequent detrimental health effects, the impact of lactation on maternal health and body composition during the reproductive years and beyond deserve attention.

If the anticipatory role for mothers is to deposit fat during pregnancy and lose it during lactation, we may ask: are mothers who do not lactate at risk for obesity? We hypothesized that repeated pregnancies in the absence of or followed by only a short period of postpartum lactation would have detrimental effects on body composition in women and increase risk for cardio-metabolic disorders in later life. To test our hypothesis, we investigated the long-term cumulative effects of repeated pregnancies and duration of postpartum breast-feeding on maternal anthropometrics, body composition and metabolic risk 16-20 years after the last parturition.

\section{Materials and methods}

\section{Study design}

The present retrospective study was part of the Calexfamily study, which has been described elsewhere ${ }^{(20,21)}$. Briefly, the study was conducted in the city of Jyväskylä and its surroundings in Central Finland in 2007 to 2008. Medical history and lifestyle factors, such as level of education, previous (stages of life: 20-29, 30-39, 40-49, $>50$ years of age) and current participation in leisuretime physical activity, and information on breast-feeding, number of pregnancies and biological children, and pre-pregnancy weight and height, were collected via a self-administered questionnaire. Participants were also asked to provide detailed information on weight change patterns during each pregnancy from their personal maternity tracking records, which have been issued to mothers since the socialization of maternal health care in
Finland. Intakes of total energy and energy-yielding nutrients were assessed from food records ${ }^{(22)}$. Breastfeeding was expressed in total exclusive (giving the infant no food or liquid other than breast milk) and partial (giving the infant some breast milk in addition to other liquid or solid foods) breast-feeding months.

The recruitment of the study population is presented schematically in Fig. 1. Briefly, 212 mothers (mean age 48, range 36-60 years) participated in the body composition and other laboratory assessments. Of this number, 206 women provided valid information on the number of biological children they had. Thirteen (6.3\%) women reported having one child, seventy-one $(34 \cdot 5 \%)$ had two, seventy-two $(35 \cdot 0 \%)$ had three, thirty-five $(17 \cdot 0 \%)$ had four and fifteen $(7 \cdot 3 \%)$ had more than four biological children. Participants were excluded if they had gestational diabetes or hypertension, were currently pregnant or reported being pregnant within 5 years before the present measurements. In addition, we excluded those who reported twin pregnancies $(n 5)$ or did not have body composition data ( $n$ 3). Thus, the final sample consisted of 198 mothers.

The 198 participants were divided into three groups according to the average duration of breast-feeding they reported (total months of breast-feeding divided by the number of biological children). WHO recommends 6 months for a minimum duration to breast-feed ${ }^{(7)}$. The lowest cut-off point was selected on the basis of this recommendation. Sixty-seven (34\%) mothers reported a short duration of breast-feeding (SDB, defined as breastfeeding their infants for less than 6 months), sixty-eight (34\%) reported a medium duration of breast-feeding (MDB, defined as breast-feeding their infants for more than 6 months but less than 10 months) and sixty-three (32\%) reported a long duration of breast-feeding (LDB, defined as breast-feeding for longer than 10 months). Five (7\%) of the SDB and two (3\%) of the LDB mothers

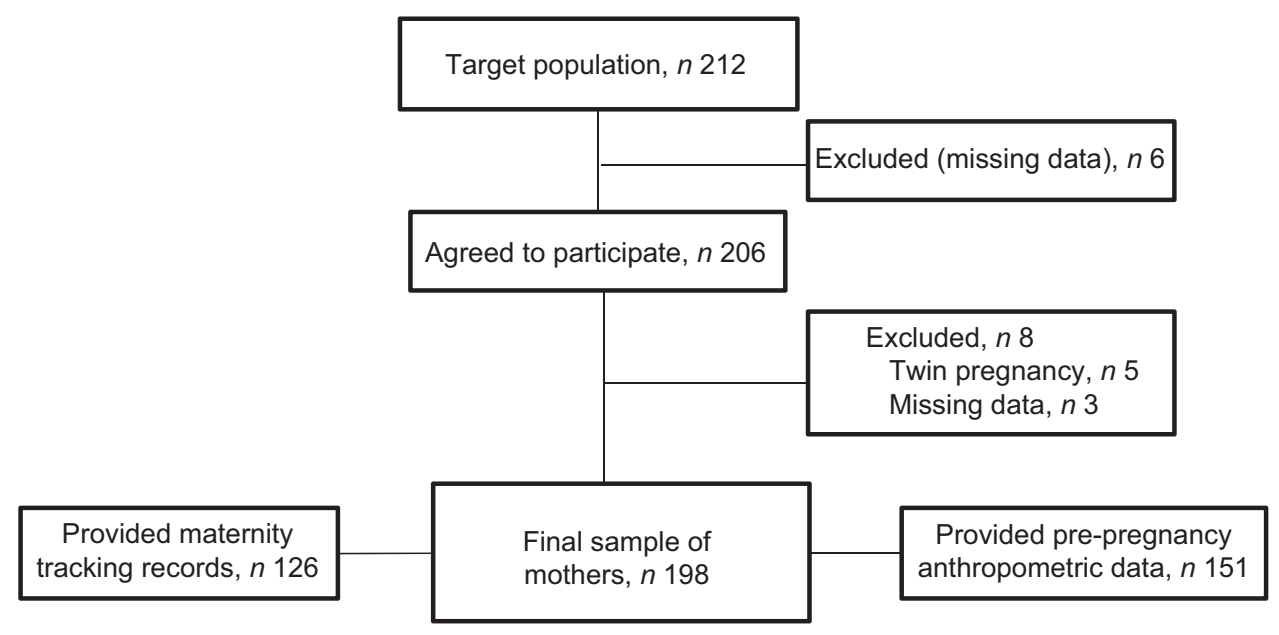

Fig. 1 The mothers (age 36-60 years) were recruited from the city of Jyväskylä and its surroundings in Central Finland, 2007-2008. After applying the inclusion criteria, the final sample consisted of 198 women 
reported that they had never exclusively breast-fed their infants. One hundred and fifty-one mothers provided prepregnancy anthropometric data and 126 mothers provided maternity tracking records. The study protocol was approved by the ethical committee of the Central Health Care District, Central Finland. Written informed consent was given by all participants prior to the assessments.

\section{Antbropometric and body composition assessments}

Body height $(\mathrm{cm})$ and weight $(\mathrm{kg})$ were measured using standardized protocols, and BMI $\left(\mathrm{kg} / \mathrm{m}^{2}\right)$ was calculated. Bone mass (BM in $\mathrm{kg}$ ), lean tissue mass (LM in kg) and fat mass (FM in $\mathrm{kg}$ ) of the whole body and in different areas of the body (arm, leg, trunk, android and gynoid, femoral and gluteal regions) were assessed using dual-energy X-ray absorptiometry (Prodigy; GE Lunar Corp., Madison, WI, USA). The CV of two repeated measurements on the same day was on average $0.7 \%$ for $\mathrm{BM}, 1 \cdot 0 \%$ for $\mathrm{LM}$ and $2 \cdot 2 \%$ for FM in the present study. Blood pressure was measured twice in the morning, by the oscillometric method, after the participants had arrived at the laboratory and sat at rest for $10 \mathrm{~min}$.

\section{Blood samples and analysis}

Blood samples were drawn from the antecubical vein in the morning (07.00-09.00 hours) after overnight fasting $(12 \mathrm{~h})$. If the mothers were in the premenopausal state, the blood sample was drawn on the fifth day from the start of menstruation. Serum was separated within $30 \mathrm{~min}$, and stored at $-80^{\circ} \mathrm{C}$ until analysis. Serum glucose, total cholesterol (TC), HDL cholesterol (HDL-C) and TAG concentrations were measured by enzymatic photometry on a Kone Pro Clinical Chemistry Analyser (Thermo Clinical Labsystems Oy, Espoo, Finland) with commercial kits. LDL cholesterol (LDL-C) was calculated using the Friedewald equation $^{(23)}$. An IMMULITE Analyser (Diagnostic Products Corporation, Los Angeles, CA, USA) and an IMMULITE Insulin Kit were used for quantitative determination of insulin. The CV of two repeated measurements on the same day was on average $3 \cdot 4 \%$ for insulin.

\section{Statistical analyses}

All data were checked for normality using the ShapiroWilk $W$ test in the SPSS for Windows statistical software package version $15 \cdot 0$ (SPSS Inc., Chicago, IL, USA). If data were not normally distributed, natural logarithms were used. ANOVA with the Least Significant Difference post boc test was used to compare differences among the breastfeeding groups in pre-pregnancy anthropometrics, age at baseline and at each pregnancy, and weight change during each pregnancy. To validate the self-estimated weight before the first pregnancy, we compared the self-estimated weight with the measured weight at the beginning of the first pregnancy using Bland-Altman analysis ${ }^{(24)}$.

A general linear model was employed to compare the differences in anthropometric, body composition, serum glucose and insulin and lipid profiles among the groups 16-20 years after the last parturition. All the analyses were adjusted for relevant factors. Statistical significance was set at $P<0 \cdot 05$.

To determine which factors were associated with weight gain, total body fat mass and android fat mass, a generalized estimating equations (GEE) model was used. The following predictors were included in the model: pre-pregnancy weight and BMI, age at first pregnancy, smoking, menopause status, level of education, previous and current participation in leisure-time physical activity, current dietary energy intake, number of biological children, and duration of exclusive and total breast-feeding months. The $R^{2}$ of the GEE model was computed according to $\operatorname{Hardin}^{(25)}$, and can be interpreted as a similar measure of the proportion of the outcome variance explained by the model as that in common linear regression.

\section{Results}

The characteristics of the participants in the year before the first pregnancy are given in Table 1. No significant differences were found in age, BMI or systolic and diastolic blood pressure between the groups before the first pregnancy. Gestational durations and inter-pregnancy intervals were similar among all groups $(P>0.05$ for all,

Table 1 Participants' characteristics before the first pregnancy according to study group: women (mean age 48, range 36-60 years) from the city of Jyväskylä and surroundings in Central Finland, 2007-2008

\begin{tabular}{|c|c|c|c|c|c|c|c|c|c|}
\hline & \multicolumn{2}{|c|}{ SDB $(n 51)$} & \multicolumn{2}{|c|}{ MDB $(n 51)$} & \multicolumn{2}{|c|}{ LDB (n 49) } & \multicolumn{3}{|c|}{$P$ value } \\
\hline & Mean & $\mathrm{SD}$ & Mean & SD & Mean & SD & SDB $v . \mathrm{MDB}$ & SDB $v$. LDB & MBD $v$. LDB \\
\hline Age (years) & $26 \cdot 4$ & $4 \cdot 8$ & $26 \cdot 0$ & $3 \cdot 5$ & $25 \cdot 9$ & $3 \cdot 5$ & 0.601 & 0.485 & 0.851 \\
\hline Height (cm) & $164 \cdot 0$ & $6 \cdot 7$ & $165 \cdot 7$ & $5 \cdot 4$ & $166 \cdot 8$ & $5 \cdot 6$ & $0 \cdot 164$ & $0.024^{*}$ & $0 \cdot 351$ \\
\hline Weight (kg) & $57 \cdot 1$ & $8 \cdot 1$ & $56 \cdot 2$ & $6 \cdot 2$ & $59 \cdot 1$ & $7 \cdot 0$ & 0.501 & 0.193 & $0 \cdot 042^{*}$ \\
\hline $\mathrm{BMI}\left(\mathrm{kg} / \mathrm{m}^{2}\right)$ & $21 \cdot 2$ & $2 \cdot 3$ & $20 \cdot 4$ & $2 \cdot 0$ & $21 \cdot 2$ & $2 \cdot 1$ & $0 \cdot 101$ & 0.874 & 0.125 \\
\hline Systolic BP (mmHg)t & $118 \cdot 8$ & $9 \cdot 2$ & $117 \cdot 0$ & $10 \cdot 6$ & $119 \cdot 5$ & $10 \cdot 5$ & 0.509 & 0.794 & 0.335 \\
\hline Diastolic BP $(\mathrm{mmHg}) \dagger$ & $70 \cdot 7$ & $7 \cdot 6$ & $69 \cdot 7$ & $8 \cdot 5$ & $72 \cdot 3$ & $6 \cdot 9$ & 0.586 & 0.414 & $0 \cdot 153$ \\
\hline
\end{tabular}

SDB, short duration of breast-feeding; MDB, medium duration of breast-feeding; LDB, long duration of breast-feeding; BP, blood pressure.

*Significant at $P<0.05$.

tBlood pressure obtained at the beginning of the first pregnancy. 


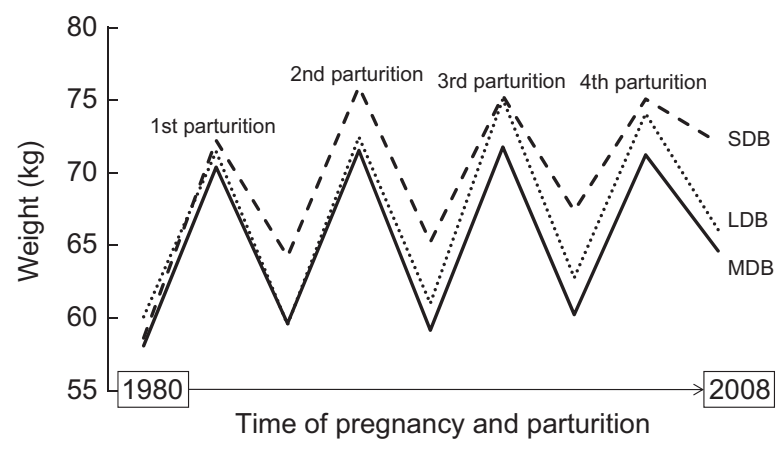

Fig. 2 ANOVA was used to compare weight change among the breast-feeding groups during the reproductive years. Body weight was similar among the groups at the beginning of the first pregnancy. After the first and each consecutive parturition, the SDB mothers retained significantly more body weight compared with MDB and LDB mothers (all $P<0.001$ ). Number of women in each group: at first pregnancy, SDB ( $n$ 38), MDB ( $n$ 44) and LDB ( $n 44)$; at second pregnancy, SDB $(n 33)$, MDB ( $n 41)$ and LDB ( $n 42)$; at third pregnancy, SDB $(n 14)$, MDB $(n 26)$ and LDB ( $n$ 30); at fourth preganancy, SDB $(n 5)$, MDB $(n 8)$ and LDB ( $n$ 15); and in year 2008, SDB ( $n 38)$, MDB ( $n 44)$ and LDB (n 39). SDB, short duration of breast-feeding; MDB, medium duration of breast-feeding; LDB, long duration of breast-feeding. Women (mean age 48, range 36-60 years) from the city of Jyväskylä and surroundings in Central Finland, 2007-2008

data not shown). Consequently, there were no significant differences in average age at the subsequent pregnancies (second pregnancy, SDB $28 \cdot 6$ (SD 3.8) years, LDB $28 \cdot 2$ $(\mathrm{SD} 3 \cdot 1)$ years and $\mathrm{MDB} 29 \cdot 0$ (SD 3.8) years; third pregnancy, SDB 31.3 (SD 4.2) years, LDB $29 \cdot 9$ (SD 3.3) years and MDB $31 \cdot 1$ (SD 3.4) years; fourth pregnancy, SDB $34 \cdot 0$ $(\mathrm{SD} 4 \cdot 3$ ) years, MDB $33 \cdot 5$ (SD $4 \cdot 3$ ) years and $\mathrm{LDB} 33 \cdot 0$ (SD $2 \cdot 1$ ) years) among the groups (all $P>0 \cdot 05$ ).

Between the years 1980 and 2008, the SDB mothers gained significantly more body weight $(14 \cdot 0(\mathrm{SD} 9 \cdot 1) \mathrm{kg})$ than either the MDB mothers $(8 \cdot 3(\mathrm{sD} 6 \cdot 5) \mathrm{kg} ; P=0 \cdot 001)$ or the LDB mothers $(7 \cdot 6$ (SD 6.6) kg; $P<0 \cdot 001$; Fig. 2). Gestational weight gains were similar among groups in all pregnancies. However, between the first parturition and the beginning of the second pregnancy, weight reduction was more significant in the $\operatorname{MDB}(-10.5(\mathrm{SD} 4.5) \mathrm{kg})$ and LDB $(-11 \cdot 4(\mathrm{SD} 3 \cdot 4) \mathrm{kg})$ mothers than in the SDB mothers $(-8 \cdot 0$ (sD $4 \cdot 4) \mathrm{kg} ; P<0 \cdot 01$ for all). In fact, only the LDB mothers had returned to their pre-pregnancy weight $(59 \cdot 5$ (SD 6.7) kg; $P=0.084$ ), whereas the MDB mothers were $1 \cdot 8$ (SD $2 \cdot 6) \mathrm{kg}$ and SDB mothers $5 \cdot 0$ (SD 4.2) $\mathrm{kg}$ heavier by the beginning of the second pregnancy $(P<0 \cdot 001$ for both). Although gradual weight gain was observed in all groups, this phenomenon followed a similar pattern in the mothers' subsequent pregnancies (Fig. 2). The selfestimated weight before the first pregnancy correlated significantly with the weight measured at the first time point (8 weeks) of the first pregnancy $\left(R^{2}=0 \cdot 898\right.$, $P<0 \cdot 001)$. Furthermore, a Bland-Altman analysis showed a good agreement (mean difference $2.6 \%$ ) and the disagreement was $7 \cdot 7 \%$ between the self-estimated and first measured weight (data not shown), regardless of the fact some weight gain is experienced during early pregnancy.

At 16-20 years after the last parturition, no significant differences between the groups in age, height, total body lean mass, total bone mass, number of biological children, smoking status, menopause, current total energy and macronutrient intake, or participation in current leisure-time physical activity were found (all $P>0 \cdot 05$; Table 2). In addition, no significant differences between the groups in previous participation in leisure-time physical activity were observed (data not shown). However, the SDB mothers weighed more and had higher BMI and total fat mass than either the MDB or LDB mothers $(P<0 \cdot 001$ for both; Table 2). In addition, a notably higher proportion of the SDB mothers had only a basic level of education compared with the MDB and LDB mothers $(P<0.05$ for both). Furthermore, the SDB mothers had significantly more fat in the android region $(46.5$ (SD 8.2)\%) than either the MDB (39.0 (SD 10.8) \%) or the LDB mothers (38.4 (SD 10.9) \%; $P<0 \cdot 01$ for both). After adjusting for level of education, the level of significance remained the same (all $P<0 \cdot 05$; Fig. 3).

In accordance with their higher fat mass, the SDB mothers had significantly higher fasting serum glucose concentration than the LDB mothers $(P=0 \cdot 033)$, and higher insulin concentrations and insulin resistance index (HOMA-IR) than either the LDB or MDB mothers (all $P<0 \cdot 05$; Table 3 ). Similarly, the SDB mothers had higher serum TC, LDL-C and TAG levels than either the LDB or MDB mothers (all $P<0 \cdot 05$ ), and lower HDL-C than the MDB mothers $(P=0 \cdot 016$; Table 3$)$. Furthermore, the SDB mothers had higher systolic and diastolic blood pressure than either the MDB or LDB mothers. After adjusting for level of education, the level of significance remained unchanged ( $P<0.05$ for both).

A GEE model was used to evaluate the risk factors associated with body composition. We found that a common predictor for low gain in body weight, total fat mass and android fat mass was long duration of breastfeeding (explaining 20\%, 24\% and $14 \%$ of the variance, respectively; Table 4). Specific predictors for high total weight gain were high age at first pregnancy (11\%), menopause $(7 \%)$ and short duration of exclusive breastfeeding $(6 \%)$. Significant predictors for high total fat mass were high pre-pregnancy weight, menopause, short duration of exclusive breast-feeding and low current leisure-time physical activity (explaining 16\%, 5\%, 11\% and $7 \%$ of the variance, respectively). Specific predictors for high android fat mass were high pre-pregnancy BMI (11\%), menopause (9\%), high age at first pregnancy (7\%) and short duration of exclusive breast-feeding (5\%).

\section{Discussion}

In the current retrospective study, we found that body weight and body composition during the reproductive 
Table 2 Participants' characteristics at 16-20 years after the last parturition according to study group: women (mean age 48, range 36-60 years) from the city of Jyväskylä and surroundings in Central Finland, 2007-2008

\begin{tabular}{|c|c|c|c|c|c|c|c|c|c|}
\hline & \multicolumn{2}{|c|}{ SDB $(n 67)$} & \multicolumn{2}{|c|}{$\operatorname{MDB}(n 68)$} & \multicolumn{2}{|c|}{ LDB $(n 62)$} & \multicolumn{3}{|c|}{$P$ value } \\
\hline & Mean & SD & Mean & SD & Mean & SD & SDB $v . M D B$ & SDB $v$. LDB & MBD $v$. LDE \\
\hline Age (years) & $47 \cdot 7$ & $5 \cdot 6$ & $48 \cdot 5$ & $4 \cdot 7$ & $48 \cdot 2$ & $4 \cdot 4$ & 0.385 & 0.629 & $0 \cdot 714$ \\
\hline Height $(\mathrm{cm})$ & $164 \cdot 1$ & $6 \cdot 1$ & $165 \cdot 3$ & $5 \cdot 5$ & $166 \cdot 0$ & $6 \cdot 2$ & 0.235 & 0.072 & 0.520 \\
\hline Weight $(\mathrm{kg}) \dagger$ & $73 \cdot 5$ & $15 \cdot 6$ & $66 \cdot 8$ & $10 \cdot 2$ & $67 \cdot 6$ & $9 \cdot 5$ & $0.002^{*}$ & $0.007^{*}$ & $0 \cdot 704$ \\
\hline BMI $\left(\mathrm{kg} / \mathrm{m}^{2}\right) \dagger$ & $27 \cdot 3$ & 5.5 & $24 \cdot 4$ & $3 \cdot 7$ & $24 \cdot 6$ & $3 \cdot 3$ & $<0.001^{\star}$ & $0.001^{*}$ & 0.847 \\
\hline Total fat mass $(\mathrm{kg}) \dagger$ & $29 \cdot 4$ & $11 \cdot 1$ & $23 \cdot 3$ & $7 \cdot 8$ & $24 \cdot 6$ & $9 \cdot 6$ & $<0.001^{*}$ & $0.006^{*}$ & 0.425 \\
\hline Total bone mass $(\mathrm{kg})$ & $2 \cdot 6$ & $0 \cdot 4$ & $2 \cdot 6$ & 0.4 & $2 \cdot 6$ & 0.4 & 0.268 & $0 \cdot 617$ & $0 \cdot 113$ \\
\hline Total lean mass (kg) & $41 \cdot 3$ & $5 \cdot 0$ & $40 \cdot 4$ & $4 \cdot 0$ & $41 \cdot 6$ & $4 \cdot 3$ & 0.216 & 0.731 & $0 \cdot 118$ \\
\hline Diastolic BP (mmHg) & 86 & 10 & 78 & 10 & 80 & 12 & $0.002^{*}$ & $0.012^{*}$ & 0.578 \\
\hline Systolic BP (mmHg) & 142 & 19 & 129 & 14 & 131 & 18 & $0.003^{*}$ & $0.032^{*}$ & 0.427 \\
\hline Smoking (no/yes; \%) & \multicolumn{2}{|c|}{$62 / 38$} & \multicolumn{2}{|c|}{$68 / 32$} & \multicolumn{2}{|c|}{$78 / 22$} & 0.495 & $0 \cdot 180$ & 0.223 \\
\hline Menopause (no/yes; \%) & \multirow{2}{*}{\multicolumn{2}{|c|}{$\begin{array}{l}80 / 20 \\
82 / 18\end{array}$}} & \multicolumn{2}{|c|}{$84 / 16$} & \multicolumn{2}{|c|}{$88 / 12$} & 0.568 & 0.239 & 0.523 \\
\hline HRT (yes/no; \%) & & & \multirow{2}{*}{\multicolumn{2}{|c|}{$\begin{array}{l}84 / 16 \\
35 / 65\end{array}$}} & \multicolumn{2}{|c|}{$84 / 16$} & $0 \cdot 760$ & 0.741 & 0.971 \\
\hline Education $(\mathrm{B} / \mathrm{H} ; \%)$ & \multicolumn{2}{|c|}{$\begin{array}{l}82 / 18 \\
56 / 44\end{array}$} & & & & & 0.015 & 0.031 & 0.746 \\
\hline $\mathrm{PA}$ (h/week) & $2 \cdot 4$ & $1 \cdot 8$ & $2 \cdot 7$ & 1.9 & $2 \cdot 1$ & $1 \cdot 7$ & 0.345 & 0.428 & 0.088 \\
\hline Biological children & $2 \cdot 7$ & $1 \cdot 0$ & $2 \cdot 9$ & $1 \cdot 3$ & $3 \cdot 0$ & 1.5 & $0 \cdot 249$ & $0 \cdot 110$ & 0.633 \\
\hline TBF per child (months) & $4 \cdot 1$ & $1 \cdot 7$ & $8 \cdot 4$ & $1 \cdot 1$ & $13 \cdot 7$ & $3 \cdot 2$ & $<0 \cdot 001^{*}$ & $<0.001^{*}$ & $<0.001^{*}$ \\
\hline Protein (\%E) & $18 \cdot 5$ & $3 \cdot 0$ & $18 \cdot 5$ & 3.5 & $18 \cdot 2$ & $2 \cdot \overline{8}$ & 0.874 & 0.493 & 0.596 \\
\hline Carbohydrates (\%E) & $45 \cdot 5$ & $6 \cdot 3$ & $45 \cdot 6$ & $6 \cdot 8$ & $46 \cdot 7$ & $5 \cdot 4$ & 0.902 & $0 \cdot 297$ & $0 \cdot 360$ \\
\hline Fats $(\% \mathrm{E})$ & $33 \cdot 7$ & $5 \cdot 2$ & $33 \cdot 4$ & $6 \cdot 3$ & 33.5 & $4 \cdot 3$ & 0.707 & 0.808 & 0.907 \\
\hline Dietary energy $(\mathrm{kJ})$ & 7385 & 1481 & 7217 & 1661 & 7075 & 1594 & 0.551 & $0 \cdot 291$ & 0.636 \\
\hline Dietary energy (kcal) & 1765 & 354 & 1725 & 397 & 1691 & 381 & 0.551 & 0.291 & 0.636 \\
\hline
\end{tabular}

SDB, short duration of breast-feeding; MDB, medium duration of breast-feeding; LDB, long duration of breast-feeding; BP, blood pressure; HRT, hormone replacement therapy; $\mathrm{B}$, basic education (high-school or upper secondary level); $\mathrm{H}$, higher education (polytechnic/academic level); PA, physical activity; TBF, total breast-feeding months; \%E, percentage of daily energy intake.

Results are adjusted for level of education.

${ }^{*}$ Significant at $P<0.05$.

tSignificance from natural log values comparison.

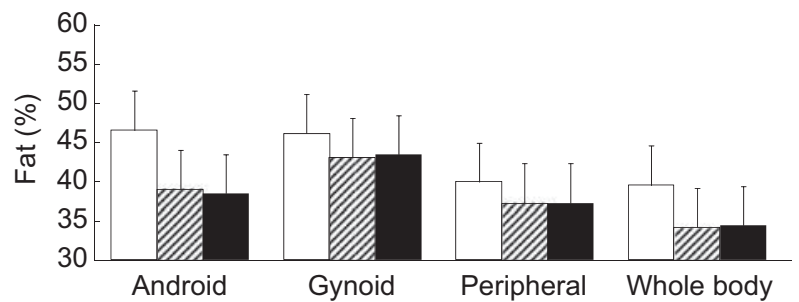

Fig. 3 A general linear model was performed to assess the difference in segmental body fat distribution 16-20 years after the last parturition among women with different durations of breast-feeding. Mothers in the SDB group ( $\square, n$ 67) had a significantly higher percentage of fat in the total body and in the android $(P<0.01$ for both), gynoid and peripheral regions $(P<0.05$ for both) than mothers in either the MDB group (שू, $n 68$ ) or the LDB group $(\square, n 62)$. Results are adjusted for level of education. Values are means with their standard deviations represented by vertical bars. SDB, short duration of breast-feeding; MDB, medium duration of breast-feeding; LDB, long duration of breast-feeding; peripheral, arms plus legs. Women (mean age 48, range 36-60 years) from the city of Jyväskylä and surroundings in Central Finland, 2007-2008

years were associated with the duration of postpartum lactation. Mothers who had breast-fed their infants for less than 6 months had higher total and relative body fat mass, particularly in the android region, compared with mothers who had breast-fed for longer than 6 months. This difference was observed 16-20 years after the last parturition and was independent of pre-pregnancy weight and BMI, menopausal status, smoking status, level of education, participation in previous and present leisuretime physical activity, and current dietary energy intake. Short duration of breast-feeding was also associated with increased risk of metabolic disorders manifested by higher fasting serum glucose concentration and insulin resistance, serum TAG, LDL-C and TC concentrations, as well as higher systolic and diastolic blood pressures.

In theory, weight loss is supported by negative energy balance due to either increased energy expenditure or reduced energy intake, or both. However, we did not find differences in current dietary energy or energy-yielding nutrient intake, or in previous or current participation in leisure-time physical activity, among the different breastfeeding duration groups. Although postpartum lactation significantly increases energy expenditure due to the production of milk in the mammary glands ${ }^{(1)}$, it is also accompanied by increased energy intake ${ }^{(15)}$. Unfortunately, we were unable to obtain postpartum dietary data from our participants. However, we speculate that given their long duration of breast-feeding and short-spaced pregnancies, it is unlikely that the participants had tried to lose weight postpartum by restricting their dietary energy intake. This indicates that postpartum weight loss cannot be explained merely by changes in energy expenditure or energy intake alone. Thus, we speculate that postpartum weight loss may relate mainly to hormonal/metabolic changes induced by lactation. 
Table 3 Laboratory test results of the participants at 16-20 years after the last parturition according to study group: women (mean age 48, range 36-60 years) from the city of Jyväskylä and surroundings in Central Finland, 2007-2008

\begin{tabular}{|c|c|c|c|c|c|c|c|c|c|}
\hline & \multicolumn{2}{|c|}{ SDB $(n 67)$} & \multicolumn{2}{|c|}{ MDB ( $n$ 68) } & \multicolumn{2}{|c|}{ LDB ( $n$ 62) } & \multicolumn{3}{|c|}{$P$ value } \\
\hline & Mean & $\mathrm{SD}$ & Mean & SD & Mean & $\mathrm{SD}$ & SDB $v . \mathrm{MDB}$ & SDB $v$. LDB & MBD $v$. LDB \\
\hline $\mathrm{TC}(\mathrm{mmol} / \mathrm{l})$ & $5 \cdot 5$ & 0.9 & $5 \cdot 5$ & 0.9 & $5 \cdot 2$ & 0.6 & $0 \cdot 811$ & $0 \cdot 015^{\star}$ & $0 \cdot 027^{\star}$ \\
\hline HDL-C (mmol/l) & $1 \cdot 6$ & 0.5 & $1 \cdot 8$ & 0.5 & $1 \cdot 7$ & 0.4 & $0.018^{\star}$ & $0 \cdot 119$ & $0 \cdot 442$ \\
\hline LDL-C (mmol/l) & $3 \cdot 4$ & $0 \cdot 7$ & $3 \cdot 2$ & 0.8 & $3 \cdot 0$ & $0 \cdot 7$ & 0.410 & $0 \cdot 047^{*}$ & 0.225 \\
\hline TAG $(\mathrm{mmol} / \mathrm{l})^{*}$ & $1 \cdot 3$ & 0.6 & $1 \cdot 0$ & 0.4 & $1 \cdot 0$ & 0.5 & $0.015^{\star}$ & $0.005^{\star}$ & 0.648 \\
\hline GLUC (mmol/l) & $5 \cdot 4$ & 0.6 & $5 \cdot 4$ & 0.8 & $5 \cdot 2$ & 0.4 & $0 \cdot 753$ & $0.029^{\star}$ & 0.059 \\
\hline INS $(\mu U / I) \dagger$ & $8 \cdot 6$ & $5 \cdot 0$ & $6 \cdot 3$ & $4 \cdot 3$ & $6 \cdot 1$ & $4 \cdot 0$ & $0.002^{*}$ & $0.002^{*}$ & $0 \cdot 898$ \\
\hline HOMA-IR† & $2 \cdot 1$ & $1 \cdot 3$ & $1 \cdot 5$ & $1 \cdot 1$ & 1.5 & $1 \cdot 1$ & $0.002^{*}$ & $0.002^{*}$ & 0.972 \\
\hline
\end{tabular}

TC, total serum cholesterol; HDL-C, HCL cholesterol; LDL-C, LDL cholesterol; GLUC, serum fasting glucose; INS, serum fasting insulin; HOMA-IR, homeostasis model assessment - insulin resistance index.

Results were adjusted for level of education.

*Significant at $P<0.05$.

tSignificance from natural log values comparison.

Table 4 Generalized estimating equations model accounting for variance in total weight gain, total fat mass and android fat mass among women (mean age 48, range 36-60 years) from the city of Jyväskylä and surroundings in Central Finland, 2007-2008

\begin{tabular}{llrr}
\hline Dependent variable & \multicolumn{1}{c}{ Predictors } & \multicolumn{1}{c}{$R^{2}$} & $P$ value \\
\hline Total weight gain & TBF & -0.20 & $<0.001$ \\
& Age at first pregnancy & -0.11 & 0.002 \\
& Menopause & -0.07 & 0.013 \\
Total fat mass & EBF & -0.06 & 0.024 \\
& TBF & -0.24 & $<0.001$ \\
& Pre-pregnancy weight & -0.16 & $<0.001$ \\
& Exclusive BF & -0.11 & 0.002 \\
& LTPA & -0.07 & 0.014 \\
& Age at first pregnancy & -0.06 & 0.031 \\
& Pre-pregnancy BMI & -0.05 & 0.049 \\
& Menopause & -0.05 & 0.035 \\
& TBF & -0.14 & $<0.001$ \\
& Pre-pregnancy BMI & -0.11 & 0.002 \\
& Menopause & -0.09 & 0.006 \\
& Age at first pregnancy & -0.07 & 0.012 \\
& Pre-pregnancy weight & -0.05 & 0.043 \\
& EBF & -0.05 & 0.035 \\
\hline
\end{tabular}

TBF, total duration of breast-feeding; EBF, exclusive breast-feeding; LTPA, leisure-time physical activity.

Only statistically significant $(P<0.05)$ predictors are presented in the table.

Indeed, after parturition, withdrawal of progesterone and the suckling of the breast by the infant facilitate the release of prolactin, thereby decreasing the level of oestrogen $^{(1)}$, which in turn enhances the mobilization of adipose tissue stores ${ }^{(26)}$. Furthermore, since prolactin also inhibits lipogenesis ${ }^{(27)}$ and suppresses glucose uptake in adipose tissue ${ }^{(28)}$, it is conceivable that the pregnancyinduced pattern of fat deposition may be reversed during lactation by the fluctuating web of hormones. Unfortunately, due to the retrospective design of our study, we were unable to obtain data on changes in the hormonal milieu during and after pregnancy that would have allowed us to explore the underlying metabolism and confirm that the hormonal changes were induced by lactation. Nevertheless, we found that short duration of breast-feeding was the strongest independent predictor for high weight gain as well as total and central fat mass accumulation 16-20 years after the last parturition, explaining 14-24\% of the variance. Therefore, the longlasting effect of hormonal changes induced by lactation on fat accumulation cannot be ruled out.

Accumulation of fat mass, especially in the android region, is associated with high risk of chronic diseases such as type 2 diabetes and $\mathrm{CVD}^{(6)}$. The present study shows the long-term effects of breast-feeding duration not only on body weight and body composition but also on blood glucose and the lipid profile. Short duration of breast-feeding was associated with long-term accumulation of cardio-metabolic risks, suggesting that proper duration of lactation may not only reverse gestational hyperlipidaemia and impaired glucose metabolism, but also stabilize it thereafter, possibly even providing protection against type 2 diabetes in later life ${ }^{(29)}$. Furthermore, most previous studies have only investigated the effect of breast-feeding in single pregnancies ${ }^{(13-16,30-32)}$, and therefore the plausible cumulative effect of weight gain and duration of breast-feeding in repeated pregnancies over time has been overlooked or neglected. In our study we found that body weight accumulated with each consecutive full-term pregnancy and that after each pregnancy women with short duration of breast-feeding gained more weight than women in the other groups. This indicates that prolonged breast-feeding may be essential for prevention of weight gain during reproductive age in women, thereby reducing the high risk for chronic diseases in later life.

Apart from duration of breast-feeding, several other factors have also been found to predict fat mass accumulation and weight retention postpartum. In our study cohort we found no differences in BMI, blood pressure or age at pre-pregnancy. However, we found that weight gain was associated with age at first pregnancy and menopausal status. Accumulation of fat mass was also moderately associated with current participation in leisuretime physical activity in addition to age at first pregnancy and menopausal status. Furthermore, mothers with the shortest duration of breast-feeding had the lowest level of 
education, suggesting that to meet the current breastfeeding recommendations enhanced counselling is needed in particular for women with a low level of education.

The strength of our study is that we were able to obtain detailed and accurate data on weight change during each pregnancy from the maternal tracking records. In addition, we were able to use data with multiple factors to assess whole body composition, including bone, muscle and fat distribution in different body compartments. However, the study has some limitations. First, breastfeeding data collected retrospectively, particularly after a considerable period of time, may be subject to recall bias. However, the limited evidence available suggests that maternal recall does provide accurate estimates of initiation and duration of breast-feeding with high validity ${ }^{(33)}$ even after 20 years $^{(34)}$. Second, it is difficult to establish the exact physiological mechanisms that drive changes of maternal body composition during the reproductive period on account of the array of potentially confounding factors. Furthermore, we did not obtain maternal tracking records and pre-pregnancy data from all of our participants. However, there were no differences in body weight between those who provided the pre-pregnancy anthropometrics and those who did not at 16-20 years after the first pregnancy. Given that weight gain pattern is heavily dependent on accurate estimation of the pre-pregnancy weight, we validated the self-estimated pre-pregnancy weight with the documented maternity tracking records. Our results showed a good agreement between the selfestimated weight and measured weight. Considering the consistency and the power of our results, the missing data did not change the paradigm of the study, which partially justifies the authenticity of the results.

In conclusion, our results provide an important public health message that short duration of breast-feeding may induce weight retention and fat mass accumulation, resulting in increased risk of cardio-metabolic disorders in later life. Encouraging women to prolong breast-feeding beyond 6 months may provide an important strategy for reducing the growing obesity epidemic and obesityrelated cardio-metabolic disorders in women.

\section{Acknowledgements}

Sources of funding: This study was financially supported by the Academy of Finland, Ministry of Education of Finland, University of Jyväskylä, Juho Vainio Foundation, and ASBMR Bridge Funding Research Grant 2006. Conflict of interest: All authors declared no conflicts of interest. The study sponsors played no role in the study design, data collection, analysis and interpretation, writing of the report, or in the decision to submit the paper for publication. The authors were solely responsible for writing and submitting the manuscript for publication. Author contributions: S.-L.C. has full access to all of the data in the study and takes full responsibility for the integrity of the data and for the accuracy of the data analysis. Study concept and design: S.-L.C., P.W., L.-T.X., M.A., S.K.-K. Acquisition of data: S.-M.C., P.W., L.-T.X., A.L., J.S., Q.W., E.V., E.M., S.-L.C. Analysis and interpretation of data: P.W., L.-T.X., A.L., J.S., Q.W., E.V., E.M., S.-M.C., M.A., S.K.-K., S.-L.C. Drafting of the manuscript: P.W., L.-T.X., Q.W., M.A., S.K.-K., S.-L.C. Critical revision of the manuscript for important intellectual content: P.W., L-T.X., A.L., J.S., Q.W., E.V., E.M., S.-M.C., M.A., S.K.-K., S.-L.C. Statistical expertise: P.W., L.-T.X., Q.W., S.-M.C. Obtained funding: M.A., S.K.-K., S.-L.C. Administrative, technical or material support: P.W., Q.X., A.L., J.S., Q.W., E.V., E.M., S.-M.C., M.A., S.K.-K., S.-L.C. Acknowledgments: The authors would like to thank the entire research staff, and especially Heli Vertamo and Erkki Helkala, for their valuable work and technical assistance on this project and all participants for their support.

\section{References}

1. Butte NF \& Hopkinson JM (1998) Body composition changes during lactation are highly variable among women. J Nutr 128, 2 Suppl., 381S-385S.

2. Zafon C (2007) Oscillations in total body fat content through life: an evolutionary perspective. Obes Rev 8, 525-530.

3. Magee LA, Abalos E, von Dadelszen P et al. (2009) Control of hypertension in pregnancy. Curr Hypertens Rep 11, 429-436.

4. Lain KY \& Catalano PM (2007) Metabolic changes in pregnancy. Clin Obstet Gynecol 50, 938-948.

5. Butte NF, Garza C, Stuff JE et al. (1984) Effect of maternal diet and body composition on lactational performance. $\mathrm{Am}$ J Clin Nutr 39, 296-306.

6. Despres JP \& Lemieux I (2006) Abdominal obesity and metabolic syndrome. Nature 444, 881-887.

7. World Health Organization/UNICEF (1990) The Innocenti Declaration on the Protection, Promotion and Support of Breastfeeding. Geneva: WHO/UNICEF.

8. Erkkola M, Salmenhaara M, Kronberg-Kippila C et al. (2010) Determinants of breast-feeding in a Finnish birth cohort. Public Health Nutr 13, 504-513.

9. Gartner LM, Morton J, Lawrence RA et al. (2005) Breastfeeding and the use of human milk. Pediatrics 115, 496-506.

10. Yngve A \& Sjostrom M (2001) Breastfeeding in countries of the European Union and EFTA: current and proposed recommendations, rationale, prevalence, duration and trends. Public Health Nutr 4, 631-645.

11. Jen KL, Juuhl N \& Lin PK (1988) Repeated pregnancy without lactation: effects on carcass composition and adipose tissue cellularity in rats. J Nutr 118, 93-98.

12. Zhong S, Almario R, Dubrinsky M et al. (1990) Repeated pregnancy without lactation: effects on maternal glycemic control, pregnancy outcome, carcass composition, and fat distribution in rats. Metabolism 39, 1127-1132.

13. Hatsu IE, McDougald DM \& Anderson AK (2008) Effect of infant feeding on maternal body composition. Int Breastfeed J 3, 18.

14. Baker JL, Gamborg M, Heitmann BL et al. (2008) Breastfeeding reduces postpartum weight retention. $\mathrm{Am} \mathrm{J}$ Clin Nutr 88, 1543-1551.

15. Dewey KG, Heinig MJ \& Nommsen LA (1993) Maternal weight-loss patterns during prolonged lactation. Am J Clin Nutr 58, 162-166. 
16. Dugdale AE \& Eaton-Evans J (1989) The effect of lactation and other factors on post-partum changes in body-weight and triceps skinfold thickness. Br J Nutr 61, 149-153.

17. Boardley DJ, Sargent RG, Coker AL et al. (1995) The relationship between diet, activity, and other factors, and postpartum weight change by race. Obstet Gynecol 86, 834-838.

18. Flegal KM, Carroll MD, Ogden CL et al. (2010) Prevalence and trends in obesity among US adults, 1999-2008. JAMA 303, 235-241.

19. Kautiainen S, Koivisto AM, Koivusilta L et al. (2009) Sociodemographic factors and a secular trend of adolescent overweight in Finland. Int J Pediatr Obes 4, 360-370.

20. Cheng S, Volgyi E, Tylavsky FA et al. (2009) Trait-specific tracking and determinants of body composition: a 7-year follow-up study of pubertal growth in girls. BMC Med 7, 5 .

21. Xu L, Nicholson P, Wang QJ et al. (2010) Fat mass accumulation compromises bone adaptation to load in Finnish women - a cross-sectional study spanning three generations. J Bone Miner Res 25, 2341-2349.

22. Lyytikainen A, Lamberg-Allardt C, Kannas L et al. (2005) Food consumption and nutrient intakes with a special focus on milk product consumption in early pubertal girls in Central Finland. Public Health Nutr 8, 284-289.

23. Friedewald WT, Levy RI \& Fredrickson DS (1972) Estimation of the concentration of low-density lipoprotein cholesterol in plasma, without use of the preparative ultracentrifuge. Clin Chem 18, 499-502.

24. Bland JM \& Altman DG (1986) Statistical methods for assessing agreement between two methods of clinical measurement. Lancet 1, 307-310.
25. Hardin JW \& Hilbe JM (2003) Generalized Estimating Equations. Boca Raton, FL: Chapman \& Hall.

26. Pansini F, Bonaccorsi G, Genovesi $F$ et al. (1990) Influence of estrogens on serum free fatty acid levels in women. J Clin Endocrinol Metab 71, 1387-1389.

27. Ben-Jonathan N, Hugo ER, Brandebourg TD et al. (2006) Focus on prolactin as a metabolic hormone. Trends Endocrinol Metab 17, 110-116.

28. Nilsson LA, Roepstorff C, Kiens B et al. (2009) Prolactin suppresses malonyl-CoA concentration in human adipose tissue. Horm Metab Res 41, 747-751.

29. Stuebe AM, Rich-Edwards JW, Willett WC et al. (2005) Duration of lactation and incidence of type 2 diabetes. JAMA 294, 2601-2610.

30. Brewer MM, Bates MR \& Vannoy LP (1989) Postpartum changes in maternal weight and body fat depots in lactating vs nonlactating women. Am J Clin Nutr 49, 259-265.

31. Janney CA, Zhang D \& Sowers M (1997) Lactation and weight retention. Am J Clin Nutr 66, 1116-1124.

32. Gigante DP, Victora CG \& Barros FC (2001) Breastfeeding has a limited long-term effect on anthropometry and body composition of Brazilian mothers. J Nutr 131, 78-84.

33. Li R, Scanlon KS \& Serdula MK (2005) The validity and reliability of maternal recall of breastfeeding practice. Nutr Rev 63, 103-110.

34. Kark JD, Troya G, Friedlander Y et al. (1984) Validity of maternal reporting of breast feeding history and the association with blood lipids in 17 year olds in Jerusalem. $J$ Epidemiol Community Health 38, 218-225. 